\title{
Charge-injection mechanisms in semiconductor nanoparticles analyzed from force microscopy experiments
}

\author{
S. Barbet, T. Mélin,* H. Diesinger, D. Deresmes, and D. Stiévenard \\ Institut d'Electronique de Microélectronique et de Nanotechnologie CNRS-UMR 8520, Département ISEN, Avenue Poincaré, \\ Boîte Postale 60069, 59652 Villeneuve d'Ascq Cedex, France \\ (Received 26 August 2005; revised manuscript received 18 October 2005; published 19 January 2006)
}

\begin{abstract}
Charge-injection and electric force microscopy experiments are performed in silicon nanoparticles deposited on a doped silicon substrate. We address in this paper the issue of the nanoparticle charging mechanisms. The nanoparticle charging is shown to occur in a regime of permanent current flow between the tip and substrate, during which the injected charge gets confined in the form of a two-dimensional electron gas at the nanoparticle-substrate interface. The equilibrium nanoparticle charge is calculated in a variational SchrödingerPoisson model in the effective mass approximation. The linearity of charge-voltage characteristics is discussed.

DOI: 10.1103/PhysRevB.73.045318 PACS number(s): 73.22. $-\mathrm{f}$, 73.90. $+\mathrm{f}$, 68.37.Ps, 81.07.Bc
\end{abstract}

\section{INTRODUCTION}

Owing to potential applications in nanoscale floating-gate memory devices, ${ }^{1}$ an increased interest has been shown in the last few years to perform charge injection experiments in semiconductor or metal nanoparticles embedded in or capped by insulators. ${ }^{2-4}$ Electric force microscopy (EFM) has appeared as a powerful tool for such studies since the microscope tip apex can be used to inject charges by addressing single nanoparticles with the biased EFM tip apex, and to detect electrostatic forces associated with stored charges by monitoring the EFM cantilever resonance frequency shifts. Charging of single nanoparticles has been reported either in systems where the nanoparticles are deposited on insulating thin films, or on conductive substrates, with an experimental sensitivity of the order of a few up to a few tens of elementary charges. The determination of the amount of stored charges from EFM data is performed either using semiquantitative $^{2,4,5}$ or quantitative ${ }^{6}$ models, or even in specific cases using the single-charge signal fluctuations. ${ }^{7}$

However, the charging mechanisms have not been debated extensively yet from force microscopy experiments. In this work, we investigate this issue both experimentally and theoretically in the case of nanoparticles deposited on a doped silicon substrate. The paper is organized as follows. In Secs. II and III, we release electrical measurements on single nanoparticles achieved by atomic force microscopy and charge injection experiments: discharging dynamics of electrons and holes, charge-voltage characteristics, and currentvoltage measurements. A model for charge injection mechanisms is then proposed based on these data, in which $(i)$ charge are transported by sequential tunneling through the tip-nanoparticle and nanoparticle-substrate oxide barriers; and (ii) the regime of permanent current flow is obtained when an equilibrium charge is stored in the nanoparticle in the form of a two-dimensional electron gas located at the nanoparticle-substrate interface. This model is developed quantitatively in Sec. IV using variational solutions of the Schrödinger and Poisson equations in the effective mass approximation. Calculated charge-voltage characteristics are compared with experiments.

\section{SAMPLES AND EXPERIMENTS}

\section{A. Samples}

A detailed description of the sample fabrication is provided in Ref. 8. We start from (100) silicon on insulator (SOI) wafers with $p$-type top and buried silicon layers of resistivity $10 \Omega \mathrm{cm}$, buried thermal oxide of thickness 400 $\mathrm{nm}$, and top silicon layer in the $\simeq 5 \mathrm{~nm}$ range [Fig. 1(a)]. Nanoparticles are achieved by thermal annealing of the SOI substrate in ultrahigh vacuum $\left(P \leqslant 10^{-10}\right.$ Torr $)$ at $T$ $=900{ }^{\circ} \mathrm{C}$ for $10 \mathrm{~min}$. During the annealing process, the SOI silicon layer breaks into an array of nanoparticles of average size $\simeq 50 \mathrm{~nm}$ and density $\simeq 30 \mu \mathrm{m}^{-2}$ [Fig. 1(b)]. After wetetching of the SOI buried oxide in hydrofluoric acid and

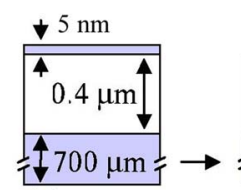

(a)

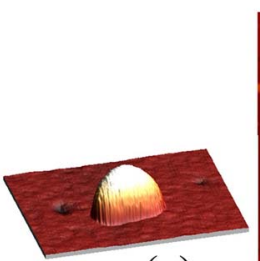

(e)

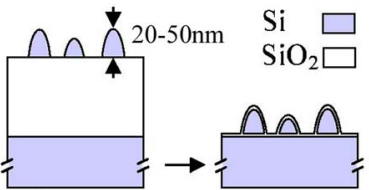

(b)

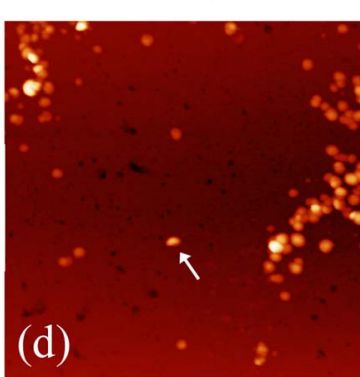

FIG. 1. (Color online) (a) Sample preparation starting from a silicon on insulator substrate with a $5 \mathrm{~nm}$ thick top layer and 400 $\mathrm{nm}$ thick buried oxide; (b) the sample is thermally annealed at $\mathrm{T}$ $=900{ }^{\circ} \mathrm{C}$ for $10 \mathrm{~min}$, leading to the formation of the silicon nanoparticles with heights in the range $\simeq 20-50 \mathrm{~nm}$; (c) nanoparticles after the buried oxide wet-etching and native oxidation; (d) atomic force microscopy image $\left(2.5 \times 2.5 \mu \mathrm{m}^{2}\right)$ of the sample. The arrow points over the nanoparticle taken for the charging experiments; (e) three-dimensional topography view of the nanoparticle. 
evaporation of the acid on a hot plate at $T \simeq 100^{\circ} \mathrm{C}$, the nanoparticles are left on the silicon substrate [see Fig. 1(c) and AFM image in Fig. 1(d)]. Since samples are stored in ambient air, a native oxide $(1-2 \mathrm{~nm})$ is formed both around the nanoparticles and on the silicon wafer. This barrier is responsible for the charge retention, ${ }^{3}$ with typical discharging time constants of the order of 5 to $10 \mathrm{~min}$.

\section{B. Charge-injection experiments, EFM and C-AFM measurements}

Charge-injection and EFM measurements have been performed using a commercial atomic force microscopy setup (Multimode/Nanoscope IIIA, Veeco Instruments) operated at atmospheric pressure under a dry nitrogen flow. We used Pt-Ir coated cantilevers with length $\simeq 200 \mu \mathrm{m}$, spring constant $1-3 \mathrm{~N} \mathrm{~m}^{-1}$, nominal resonance frequency $f_{0} \simeq 60 \mathrm{kHz}$ with typical quality factor $Q \simeq 200$.

Charge-injection experiments are achieved by pressing the EFM tip apex biased at $V_{i n j}$ with respect to the substrate on the top of a given nanoparticle. The mechanical contact is monitored by the cantilever static deflection during the charge-injection process and corresponds to a few $\mathrm{nN}$ force between the tip and nanoparticle.

Charge detection is then performed using EFM experiments, i.e., by measuring long-range Coulomb force gradients from the EFM cantilever resonance frequency shifts. Experimentally, the acquisition of EFM data is interleaved line by line into the sample topography imaging in a twopass measurement. For each scan line, the atomic force microscopy setup is first used to record the sample topography in tapping mode. EFM data are then obtained in a second pass, where the EFM tip is retracted from the sample surface, polarized at a $V_{E F M}$ voltage with respect to the substrate, and oscillated at a constant distance over the substrate plane [see Fig. 2(a)]. The typical distance between the EFM tip apex and the substrate is $z \simeq 100 \mathrm{~nm}$. EFM measurements consist in recording the cantilever resonance frequency shift $\Delta f$ with respect to its nominal value $f_{0}$ when the tip is scanned over the sample surface with $V_{E F M}$ set to zero. To do so, a feedback loop is introduced during the EFM pass over the sample to directly measure $\Delta f$ by maintaining a setpoint EFM cantilever phase $\phi_{0}$.

Conducting-atomic force microscopy ( $c$-AFM) measurements have been performed using a homemade setup with a preamplifier directly located inside the cantilever holder plate. This system was used to record current-voltage characteristics within a few seconds with a typical 15 fA noise level. Experimentally, $c$-AFM data were acquired with the EFM tip pressed on the sample in contact mode using a $\simeq 5 \mathrm{nN}$ contact force.

\section{EXPERIMENTAL RESULTS}

\section{A. Discharging dynamics}

Charge-injection experiments have been performed for negative and positive injection voltages $V_{i n j}$, leading respectively to negative and positive stored charges. The EFM data of the discharging behavior of the nanoparticle of Fig. 1(e) is

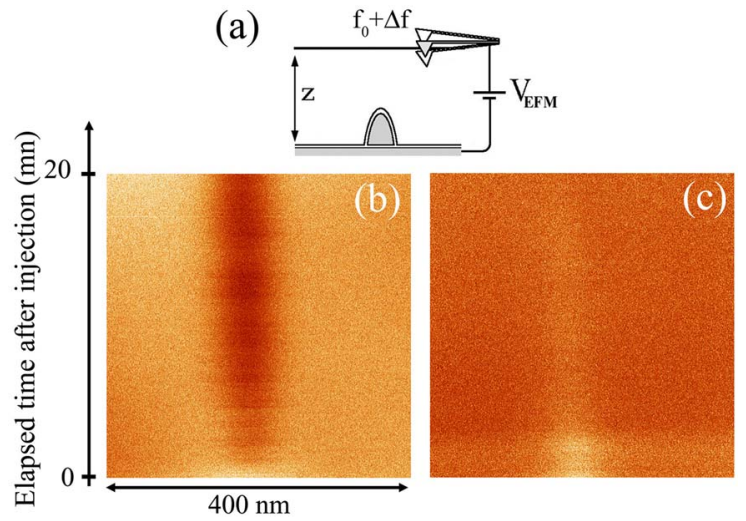

FIG. 2. (Color online) (a) Schematics of the charge detection experiment, in which the EFM cantilever is oscillated at a distance $z \simeq 100 \mathrm{~nm}$ over the substrate plane and biased at $V_{E F M}$ with respect to the substrate plane. The recorded EFM signal is the shift $\Delta f$ of the cantilever resonance frequency with respect to its nominal frequency $f_{0}$. (b) Discharging dynamics of the single particle of Fig. 1(e) with height $50 \mathrm{~nm}$. The frequency scale is $20 \mathrm{~Hz}$. Data have been recorded using $V_{E F M}=-2.5 \mathrm{~V}$ after charge injection using $V_{i n j}=-6 \mathrm{~V}$ for $30 \mathrm{~s}$. (c) Same experiment after injection experiment using $V_{i n j}=6 \mathrm{~V}$ for $30 \mathrm{~s}$, and using $V_{E F M}=2.5 \mathrm{~V}$ during detection.

shown in Fig. 2 after charge injection with opposite biases $\left(V_{i n j}= \pm 6 \mathrm{~V}\right.$ for $\left.30 \mathrm{~s}\right)$. EFM images of Fig. 2 were scanned in line mode across the nanoparticle, by stopping the $y$-axis piezoscan so that the horizontal scale corresponds to distances, while the vertical scale corresponds to the elapsed time after charging with a $20 \mathrm{mn}$ time scale. The origin of the time is taken $\simeq 30 \mathrm{~s}$ after the end of the charge-injection experiments. The two images were acquired using the same signs for $V_{E F M}$ and for $V_{i n j}$, and with an amount of stored charges high enough so that the EFM signals are dominated in both cases at $t=0$ by the repulsive interaction between the stored charges and the capacitive charges at the tip apex., This leads to the bright contrasts observed in both images at $t=0$ (positive EFM cantilever frequency shift with respect to the substrate, or, equivalently, detection of a repulsive-force gradient). In this configuration, the nanoparticle discharge corresponds to a transition from the repulsive-force gradient to the attractive-capacitive force gradient expected for the neutral nanoparticle, as explained in Ref. 3. The nanoparticle signal therefore turns from a positive frequency shift (bright feature) to a negative frequency shift (dark feature). This inversion can be clearly observed in Fig. 2(b) (negatively charged nanoparticle at $t=0)$ and occurs with a time scale $\tau_{-} \simeq 2 \mathrm{~min}$. In the case of Fig. 2(c) (positively charged nanoparticle at $t=0$ ), the decay time is much longer, with a contrast inversion occuring for $\tau_{+} \simeq 25 \mathrm{~min}$, the EFM signal remaining therefore dominated by repulsive-force gradients during the 20 min scan of Fig. 2(c).

A tentative explanation for the strong variation of the decay times for negative and positive charges is a discharge mechanism based on tunneling of the stored charge from the nanoparticle to the substrate through the $1-2 \mathrm{~nm}$ thick substrate oxide barrier. The tunneling barrier is indeed of greater amplitude for holes than for electrons, and thus leads to longer decay times for holes, as observed in Fig. 2. This 


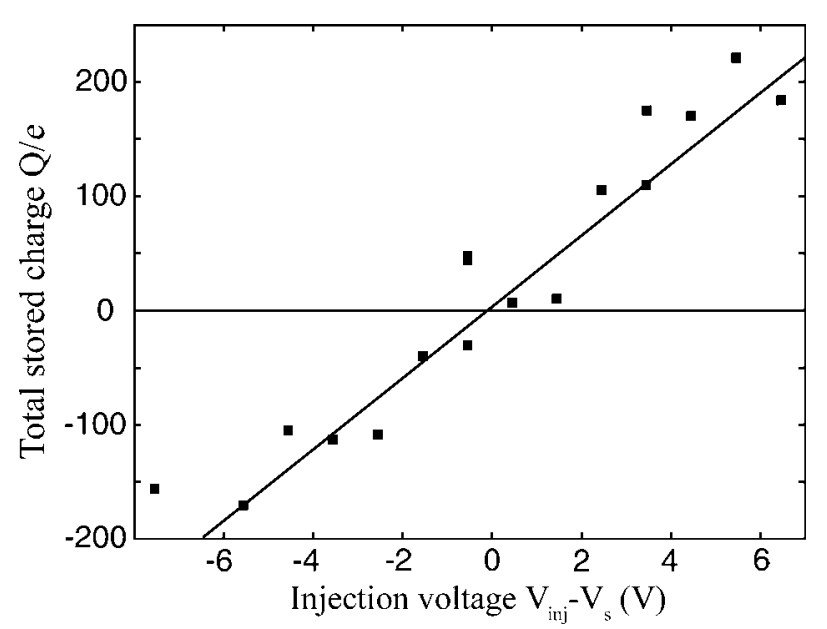

FIG. 3. Charge-voltage characteristics for the nanoparticle with $50 \mathrm{~nm}$ height of Fig. 1(e). The charge-injection experiments are those used in Ref. 9 where a constant injection time $\tau_{i n j}=30 \mathrm{~s}$ has been used. Data are plotted as a function of $V_{i n j}-V_{s}, V_{s}$ being the sample surface potential (here $V_{s}=0.55 \mathrm{~V}$ ). The line is a fit to experimental data using a linear model.

mechanism has already been pointed out in the case of CdSe nanocrystals ${ }^{7}$ separated from a conductor by an ultrathin tunnel barrier, and exhibiting a blinking between a neutral and a single positively charged state. In this case, the difference of tunneling barrier heights for holes and electrons was mentioned to account for the sign of the nanoparticle charged state, while blinking time constants were also found in the $\simeq 10$ min range.

However, an alternative explanation for the difference in decay times for negative and positive charges may originate in the doping type of the substrate ( $p$-type in our experiments). Measurements of discharging dynamics of injected electrons or holes would be required on both types of substrates to distinguish between different discharging times linked to different barrier heights, or to, e.g., depletion effects in the semiconductor substrate. This analysis, however, falls beyond the scope of the present paper.

\section{B. Charge-voltage characteristics}

Charge-voltage characteristics are obtained by repeating the charge-injection experiments on a given nanoparticle and measuring the stored charge as a function of the injection voltage $V_{i n j}$. We report in Fig. 3 the charge-injection characteristics obtained in the case of the nanoparticle of Fig. 1(e). ${ }^{10}$ Injection experiments have been performed here with a constant injection time $\tau_{i n j}=30 \mathrm{~s}$. The stored charge has been measured quantitatively using the model of Ref. 6. The main linear behavior in Fig. 3 corresponds to charge injection in the nanoparticle volume, and was proposed to originate in tunneling processes through the tip-nanoparticle and nanoparticle-substrate oxide barriers. ${ }^{12,13}$

The purpose of this paper will be to provide a quantitative analysis of the linear behavior of the charge-voltage characteristics in silicon nanoparticles. Data of Fig. 3 already reflect the physics of the charge-injection mechanims. For in-

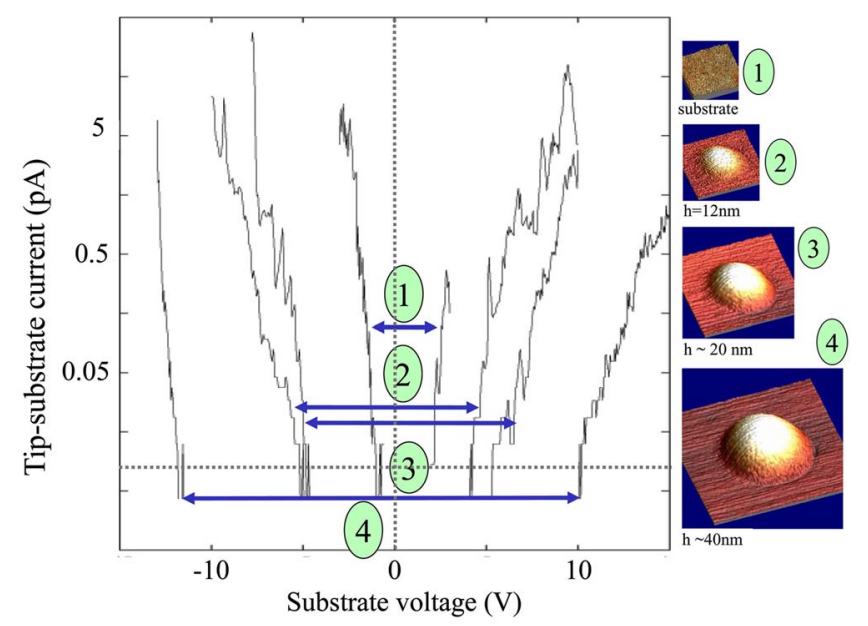

FIG. 4. (Color online) Conducting-AFM current voltage characteristics obtained with the EFM tip put on the doped semiconductor substrate native oxide layer (label 1); on nanoparticles with height $12 \mathrm{~nm}$ (label 2), $20 \mathrm{~nm}$ (label 3), and $40 \mathrm{~nm}$ (label 4). Tip currents are displayed in logarithmic scale. The voltage here has been applied to the substrate. The arrows in the $I(V)$ characteristics are a guide to the eye to show the gradual increase of the sample voltage necessary to obtain a current above the detection limit $(\simeq 15 \mathrm{fA})$ of the $c$-AFM setup.

stance, the fairly large amount of charge stored for moderate $\left(V_{i n j}<10 \mathrm{~V}\right)$ injection voltage is a consequence of the electrostatic screening induced by the conductive substrate, ${ }^{9}$ in contrast, for example, with charging experiments performed on nanocrystals deposited on dielectric thin films, ${ }^{2}$ where a few tens of electrons only can be injected for similar voltages and nanoparticle sizes. Also, from a linear fit of the data of Fig. 3, one can estimate $\mathrm{a} \simeq 25 \mathrm{meV}$ effective "charging energy" (i.e., the energy necessary to inject an extra electron into the nanoparticle). This experimental value is, however, not consistent with the actual charging energy of such a large-radius nanoparticle. It rather reflects here the mesoscopic size of the nanoparticle and the strong role played by the relaxation of injected charges in the nanoparticle. This effect will be discussed below.

\section{Current-voltage characteristics}

To gain insight into the charging mechanisms, we also performed conducting-AFM measurements on the nanoparticles. Results are given in Fig. 4. The curve labeled 1 in Fig. 4 corresponds to the current-voltage ${ }^{11}$ characteristics re- $^{-}$ corded on the sample substrate, i.e., $p$-type doped silicon covered by a native oxide. It is typical of tunneling processes from a metallized tip to a $p$-type doped semiconductor, with an easier onset of the tunneling current for negative rather than positive samples voltages. As seen from Fig. 4 the voltage zone $\Delta V_{\text {onset }}$ for which the tunneling current falls below our $c$-AFM setup detection limit (15 fA) is typically $3 \mathrm{~V}$ wide, greater than the silicon substrate band gap.

Current-voltage characteristics have then been recorded for nanoparticles with various heights (see curves labeled 2, 3 , and 4 corresponding to nanoparticles with height $12 \mathrm{~nm}$, 

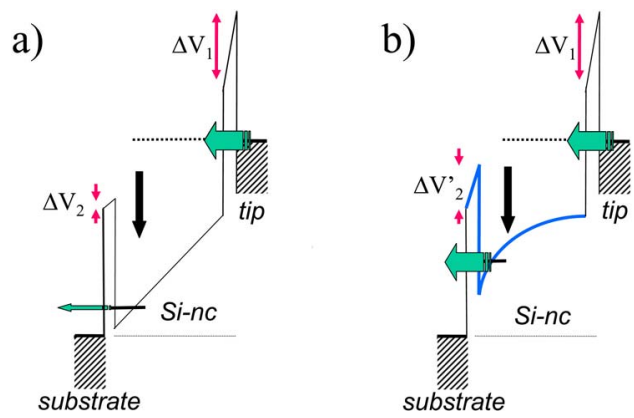

FIG. 5. (Color online) (a) Energy diagram for the tipnanoparticle and nanoparticle-substrate tunnel barriers for a neutral nanoparticle. The EFM tip is here negatively biased with respect to the substrate electrode. Due to the electric field enhancement at the EFM tip apex, the barrier potential drop $\Delta V_{1}$ at the EFM tipnanoparticle interface is greater than the barrier $\Delta V_{2}$ at the nanoparticle-substrate interface. Due to this difference, charges having relaxed (vertical arrow) inside the nanoparticle after tunneling into the nanoparticle get trapped and accumulate at the nanoparticle-substrate interface. (b) Similar energy diagram in the case of the charged nanoparticle. The band bending associated with the stored charge induces a potential rise for the stored charge, as well as an enhancement of the electric field at the nanoparticlesubstrate barrier. An equilibrium regime is found in a regime of permanence current flow when the transmission of the nanoparticlesubstrate barrier equals that of the tip-nanoparticle barrier.

$30 \mathrm{~nm}$, and $40 \mathrm{~nm})$. The extent $\Delta V_{\text {onset }}$ of the voltage zone for which the current is less than the $c$-AFM setup detection limit is seen to gradually rise with the height of the nanoparticle, and reaches $\Delta V_{\text {onset }}>20 \mathrm{~V}$ for the nanoparticle with 40 nm height.

The experimental data first show that a regime of detectable current flow can always be reached even for the bigger nanoparticles. Also, experimental data show that the higher the nanoparticle, the smaller the efficiency of the tipsubstrate voltage to pass a given current through the nanoparticle. It suggests that the current likely originates in the voltage drops across the tunnel oxide barriers at the tipnanoparticle and nanoparticle-surface interfaces, which are reduced for experiments performed on bigger size nanoparticles for a given sample voltage. This assumption is consistent with $c$-AFM measurements performed on various points across a given nanoparticle (not shown here), and showing a gradual increase of the $\Delta V_{\text {onset }}$ zone as the current-voltage characteristics is measured closer to the top of the nanoparticle (and hence with a bigger tip-substrate distance).

\section{Charge-injection mechanisms}

At this stage, a most simple model for the chargeinjection mechanisms can be derived in order to account for the experimental results of the previous sections. Assuming that the EFM tip and substrate are metal electrodes with a work function equal to the semiconductor nanoparticle affinity, the following three-step model can be proposed for the electron injection as depicted in Fig. 5(a), in which $(i)$ charges are first injected from the EFM tip into the nanoparticle by tunneling through the nanoparticle oxide at the tip- nanoparticle interface; (ii) injected charges relax inside the nanoparticle and get confined at the nanoparticle-substrate interface; (iii) trapped charges tunnel to the substrate through the substrate oxide. Since in our experiments the electric field is enhanced at the EFM tip apex, the tunnel transmission of the tip-nanoparticle barrier is much greater than that of the nanoparticle-substrate barrier. This is responsible for the charge accumulation in the nanoparticle and for a rise of the electrostatic potential at the nanoparticle-substrate interface. This potential rise increases, on the other hand, the transmission of the nanoparticle-substrate tunnel barrier, and therefore generates an upper limit for the amount of stored charge. An equilibrium is indeed found when a regime of permanent current flow is established for the transport through the tip-nanoparticle and nanoparticle-substrate barriers [see Fig. 5(b)].

This model is in qualitative agreement with our experiments.

(i) The fact that electrons (resp. holes) are injected for negative (resp. positive) EFM tip polarizations is a consequence of the greater tunnel transmission through the nanoparticle cap oxide barrier compared to the substrate oxide barrier. The longer retention time measured for holes compared to electrons is also in agreement with a charge transfer based on tunneling processes.

(ii) Experimental current-voltage characteristics are consistent with a regime of permanent current flow. Since the higher the nanoparticle, the smaller the current levels at a given bias $V_{i n j}$, current-voltage characteristics are also found in agreement with the proposed model in which the current level is determined by voltage drops at the oxide barriers, and not by the voltage drop inside the nanoparticle. This also strongly emphasizes the role of relaxation processes in the nanoparticle.

(iii) Finally, the linear behavior of the charge-voltage characteristics can be understood in a most simple manner if the nanoparticle and substrate oxides have the same thickness. In this case, to ensure an equal transmission between oxide barriers, the electrostatic potential rise $\Delta V_{Q}$ associated with the stored charge $Q$ has to compensate the difference $\Delta V_{1}-\Delta V_{2}$ of the voltage drops at interfaces [see Figs. 5(a) and 5(b)] prior to charging. Since $\Delta V_{1}-\Delta V_{2}$ is proportional to the injection bias $V_{i n j}$, and since band-bending effects in the nanoparticle are proportional to the charge $Q$, this means that $Q$ should be proportional to $V_{i n j}$ as observed experimentally.

The purpose of the following section will be to develop this model beyond qualitative considerations, taking into account the quantum confinement of electronic states at the nanoparticle-substrate interface during the charge injection experiments.

\section{MODELIZATION}

\section{A. Wave function confinement}

The quantum confinement of electronic states at the nanoparticle-substrate interface during the injection process is described in the effective-mass approximation using a onedimensional model. We define as a function of the distance $z$ 


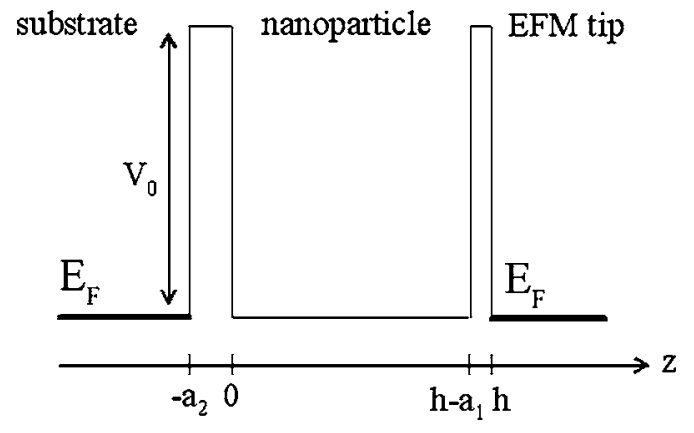

FIG. 6. One-dimensional energy diagram for the nanoparticle contacted by the EFM tip at $V_{i n j}=0 . a_{1}$ and $a_{2}$ account respectively for the nanoparticle-substrate and tip-nanoparticle oxide barriers, and $h$ for the nanoparticle height. The oxide energy barrier is $V_{0}$.

(see Fig. 6), the substrate $\left(z \leqslant-a_{2}\right)$ taken in first approximation as a metal electrode; the silicon dioxide tunnel barrier between the nanoparticle and substrate of thickness $a_{2}$ and height $V_{0}$; the silicon nanoparticle defined for $0 \leqslant z \leqslant h-a_{1}$ where $h$ is the nanoparticle height; the silicon dioxide barrier of thickness $a_{1}$ at the tip-nanoparticle interface for $h-a_{1}$ $\leqslant z \leqslant h$. In the case of the experimental data of this paper, $a_{2}=a_{1} \simeq 1-2 \mathrm{~nm}, V_{0}=4 \mathrm{eV}, h \simeq 50 \mathrm{~nm}$.

When the EFM tip is negatively biased during the chargeinjection experiment, electrons first tunnel through the tipnanoparticle dioxide barrier and get confined at the nanoparticle-substrate interface. To describe the corresponding quantum-confined electronic state, we solve the coupled Schrödinger and Poisson equations variationally using Fang and Howard wave functions for the first confined electronic state in $z,{ }^{14}$

$$
\psi_{b}(z)=\sqrt{\frac{2}{b^{3}}} z \exp (-z / b)
$$

where the variational parameter $b$ accounts for the typical confinement length of $\psi_{b}$. The Hamiltonian terms are the electron kinetic energy $H_{\text {cin }}$ corresponding to the motion in the $x$ and $y$ directions, the Hamiltonian due to the external electric field applied during the charge injection process $H_{\text {field }}=\alpha z$, and the Hamiltonian associated with the electrostatic interactions $H_{e e}$. Prior to any charging, the external field already confines the electron wave function at the nanoparticle interface described as a triangular quantum well. When charges are trapped inside the nanoparticle, it forms a two-dimensional electron gas of surface density $n_{s}$ at the nanoparticle-substrate interface [see Fig. 7(a)]. Electrostatic interactions in the case of the charged nanoparticle, however, enhance the electronic wave function confinement at the nanoparticle-substrate interface [see Fig. 7(b)] and thus need to be properly taken into account in the wave function calculation.

The average values of $H_{\text {cin }}$ and $H_{\text {field }}$ for $\psi_{b}$ lead to $\left\langle H_{\text {cin }}\right\rangle=\hbar^{2} / 2 m b^{2}$ and $\left\langle H_{\text {field }}\right\rangle=\alpha b / 2$. To compute the average electrostatic interaction $\left\langle H_{e e}\right\rangle$, the electrostatic potential $V_{e e}(z)$ is written as

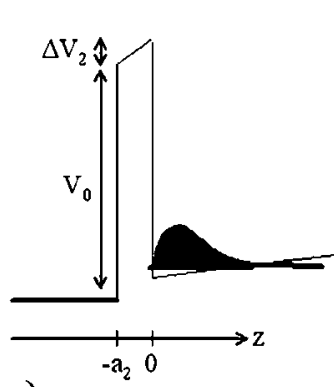

a)

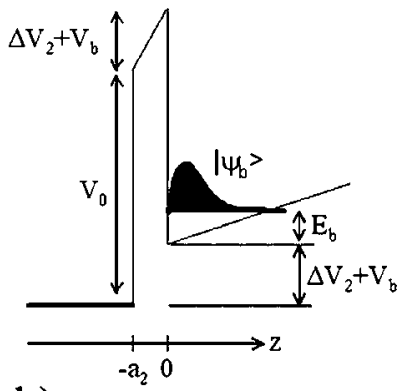

b)

FIG. 7. (a) Confinement of the electronic wave function $\psi_{b}$ at the nanoparticle-substrate interface due to the external electric field prior to the nanoparticle charging $\left(Q=0\right.$ or $\left.n_{s}=0\right)$. (b) Confinement enhancement when $Q\left(\right.$ or $\left.n_{s}\right) \neq 0$.

$$
V_{e e}(z)=\left(A z^{2}+B z+C\right) \exp (-2 z / b)+A^{\prime} z+B^{\prime},
$$

where the values for $A, B, C, A^{\prime}$, and $B^{\prime}$ are obtained by solving the Poisson equation for a given surface density $n_{s}$ for the electron gas confined at the nanoparticle-substrate interface. The determination of $V_{e e}(z)$ leads to the value of $\left\langle H_{e e}\right\rangle$, and thus to the total average energy

$$
\left\langle\psi_{b}|H| \psi_{b}\right\rangle=\frac{\hbar^{2}}{2 m b^{2}}+\frac{\alpha b}{2}+\frac{33 e^{2} n_{s} b}{32 \epsilon_{0} \epsilon_{S i}},
$$

where $\epsilon_{0}$ and $\epsilon_{S i}$ account respectively for the vacuum permittivity and the silicon nanoparticle dielectric constant. The above formula for $\left\langle\psi_{b}|H| \psi_{b}\right\rangle$ represents the average energy of the wave function $\psi_{b}$ with respect to the energy of the triangular quantum well in $z=0$. It is seen that the electrostatic interactions are proportional to $b$, such as the average energy $\psi_{b}$ in the external field. However, the field enhancement also exhibits a linear dependence with $n_{s}$, which needs to be expressed to carry out the minimization of $\left\langle\psi_{b}|H| \psi_{b}\right\rangle$ with respect to $b$.

\section{B. Tunnel currents}

A relationship between $n_{s}$ and $b$ can be derived in the regime of permanent current flow, by equalling the two tunnel currents flowing through the tip-nanoparticle and nanoparticle-substrate interfaces. Approximating the tipnanoparticle barrier as a trapezoidal tunnel barrier, the absolute magnitude of the "incoming" tunnel current is

$$
I_{i n}=K_{1} \exp \left[-\frac{2 a_{1}}{\hbar} \sqrt{2 m^{*}\left(V_{0}-\frac{\Delta V_{1}}{2}\right)}\right],
$$

where $m^{*}$ is the effective mass of the tunneling electrons, and $K_{1}$ is a constant proportional to the contact area $S_{1}$ between the tip and the nanoparticle and to the voltage drop $\Delta V_{1}$ at the tip-nanoparticle oxide interface.

The case of the "outgoing" tunnel current is more tedious, since the nanoparticle-substrate barrier can be either trapezoidal or triangular depending on the rise of the energy of $\left|\psi_{b}\right\rangle$ with respect to the substrate electrode. If the nanoparticle-substrate barrier is of trapezoidal shape, the absolute magnitude of the outgoing tunnel current $I_{\text {out }}$ reads 


$$
I_{\text {out }}=K_{2} \exp \left[-\frac{2 a_{2}}{\hbar} \sqrt{2 m^{*}\left(V_{0}-\frac{\Delta V_{2}+V_{b}}{2}-E_{b}\right)}\right],
$$

where $K_{2}$ is the tunneling prefactor at the nanoparticlesubstrate interface biased with $\Delta V_{2}+V_{b}$ when the nanoparticle is charged. $E_{b}$ is the energy level of the confined electrons measured with respect to the minimum of the triangular quantum well. $V_{b}$ and $E_{b}$ are shown schematically in Fig. 7(b). $V_{b}=a_{2} e^{2} n_{s} / \epsilon_{0} \epsilon_{S i}$ is easily obtained from the electrostatic potential $V_{e e}(z)$, and $E_{b}$ corresponds to $\left\langle\psi_{b}|H| \psi_{b}\right\rangle$.

If the nanoparticle-substrate barrier is of triangular shape, the formula for the absolute magnitude of the outgoing tunnel current is

$$
I_{\text {out }}=K_{2}^{\prime} \exp \left[-\frac{2 a_{2}^{\prime}}{\hbar} \sqrt{m^{*}\left(V_{0}-E_{b}\right)}\right],
$$

where $a_{2}^{\prime}=\left(V_{0}-E_{b}+V_{b}\right) / \Delta V_{2} \cdot a_{2}$ is the reduced effective width for electrons tunneling at the nanoparticle-substrate interface and $K_{2}^{\prime}$ is the prefactor associated with the geometry of the triangular barrier.

Finally, the energy rise of the confined electrons at the nanoparticle-substrate interface (measured with respect to the substrate Fermi level) is limited in any case, and cannot exceed the energy of electrons in the EFM tip. In this situation (i.e., for $\Delta V_{2}+V_{b}+E_{b}=-e V_{i n j}$ ), a reflection mechanism sets in for the incoming tunnel current, which is limited by the outcoming tunnel current in order to preserve a regime of permanent current through the nanoparticle interfaces.

\section{Numerical calculations and comparison with experiments}

To compute the nanoparticle charge state, numerical calculations are performed as follows.

The first step consists in calculating the voltage drops $\Delta V_{1}$ and $\Delta V_{2}$ at the tip-nanoparticle and nanoparticle-substrate interfaces prior to charging. This is done numerically, by solving the Poisson equation in a cylindrical symmetry for the EFM metal tip in contact with the top of the nanoparticle, and biased at $V_{i n j}$ with respect to the substrate considered as a metal as well. Actual geometries of the nanoparticle (height $h$, shape, aspect ratio) and of the EFM tip apex (apex radius, tip cone angle) are taken into account during this step as explained in Ref. 6, as well as the oxide thicknesses $a_{1}$ and $a_{2}$ at the tip-nanoparticle and nanoparticle-substrate interfaces.

We then compute the nanoparticle charge $Q$ using the above-described model, i.e., by calculating the surface charge density $n_{s}$ for electrons confined at the nanoparticlesubstrate interface during the charge injection experiment. To do so, minimize the value of the average energy $\left\langle\psi_{b}|H| \psi_{b}\right\rangle$ as a function of the variational parameter $b$, the relationship between the surface density $n_{s}$ and $b$ being obtained by the conservation of the incoming and outcoming tunnel currents $I_{\text {in }}$ and $I_{\text {out }}$.

We display in Fig. 8 the results of the calculation for a hemiellipsoidal nanoparticle with height $h=50 \mathrm{~nm}$ and base radius $40 \mathrm{~nm}$. The voltage drops at the tip-nanoparticle and nanoparticle-substrate oxide barriers are computed using $a_{1}$ $=a_{2}=1.8 \mathrm{~nm}$, and an EFM tip with $25 \mathrm{~nm}$ apex radius, which

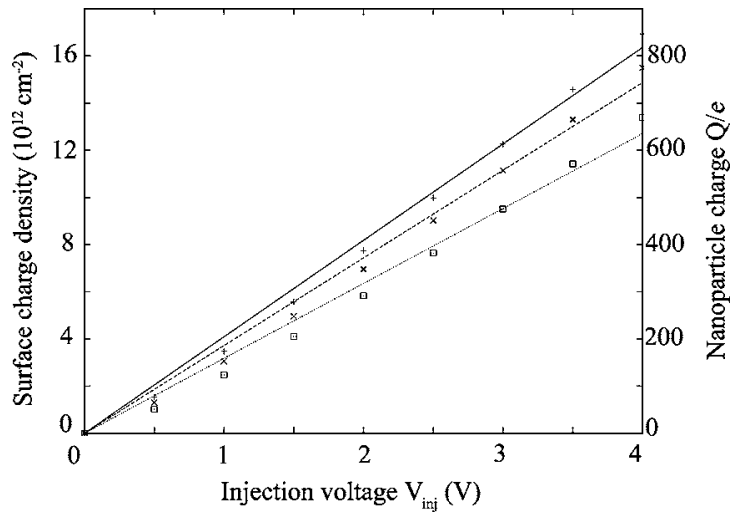

FIG. 8. Calculated surface charge density $n_{s}$ for a nanoparticle of height $h=50 \mathrm{~nm}$ and base radius $40 \mathrm{~nm}$ as a function of the injection voltage $V_{i n j}$. The corresponding nanoparticle charge $Q$ is indicated in the right vertical axis. The three sets of data correspond to calculations and the associated linear fits using an effective electron mass: $m^{*}=m_{0}(+$, linear fit in full line $), m^{*}=0.5 m_{0}(\times$, linear fit in dashed line), and $m^{*}=0.2 m_{0}(\bullet$, linear fit in dotted line).

gives $\Delta V_{1}=0.47 \mathrm{~V}$ and $\Delta V_{2}=0.025 \mathrm{~V}$ for a unit injection voltage $\left(V_{i n j}=1 \mathrm{~V}\right)$. The calculated surface charge density $n_{S}$ is then calculated and plotted as a function of the injection voltage $V_{i n j}$ in Fig. 8 for three distinct effective masses for electrons: $m^{*}=m_{0}, m^{*}=0.5 m_{0}$, and $m^{*}=0.2 m_{0}$.

Results display a quasilinear behavior as a function of the injection voltage $V_{i n j}$, in agreement with the experimental charge-voltage characteristics of Fig. 3. Moreover, the calculated charge densities exhibit only a weak dependence as a function of the electronic effective masses. ${ }^{15}$ This is consistent with the experimental observation that electrons and holes have a similar injection efficiency: no significant change of slope can be detected for negative and positive biases within experimental accuracy.

The main discrepancy between the calculated and experimental results is the amplitude of the charge densities, which is calculated to be $\simeq 7$ times higher than in experiments. Though this effect is rather large, this may be explained by the following considerations:

(i) First of all, our model assumes a total relaxation of charges injected in the nanoparticle. This is, however, not likely, as seen, for instance, from the current levels obtained on smaller $(\simeq 20 \mathrm{~nm}$ high) nanoparticles, and the typical charging times (.1 to $1 \mathrm{~s}$ ) observed for nanoparticles with similar sizes. ${ }^{12}$ In this case, charges having relaxed at the nanoparticle-substrate interface only represent a fraction of the total nanoparticle charge, consistent with the fact that calculations overestimate the amount of stored charges.

(ii) Also, the substrates used in experiments are not metallic. This certainly overestimates the amount of stored charges in the calculations by comparison with experiments, since in practice a part of the injection voltage $V_{i n j}$ drops in the substrate during the injection experiment and does not contribute to the nanoparticle charging.

(iii) Finally, the electric field at the tip-nanoparticle tunnel barrier should also be affected by the charge stored in the nanoparticle. We did not take into account this effect at the present stage of our calculations since the charge is stored at 
the nanoparticle-surface interface during the injection experiment, and primarily alters the electric field and the electron wave functions at this interface. However, the nanoparticle charging should also reduce the voltage drop $\Delta V_{1}$ at the tipnanoparticle interface and thus lower the actual nanoparticle charge. This effect, which can be computed self-consistently, will also contribute to reduce the discrepancy between experiments and theoretical calculations.

\section{CONCLUSION}

In conclusion, we addressed in this paper the issue of the charging mechanims of semiconductor nanoparticles from experimental force microscopy measurements. The nanoparticle charging was shown to occur in a regime of permanent current flow between the tip and substrate if nanoparticles are deposited on a conductive substrate. The equilibrium nanoparticle charge has been calculated in a variational Schrödinger-Poisson model in the effective mass approximation, showing that the stored charge is confined during the injection experiment in the form of a two-dimensional electron gas at the nanoparticle-substrate interface. The principles of this model can be extended to the case of chargeinjection experiments of nanoparticles deposited on insulators, where a null current is established at equilibrium.

\section{ACKNOWLEDGMENTS}

We are indebted to J. P. Lansun and C. Delerue for technical help and fruitful discussions. This work was supported by an ACI Nanostructures grant from the French Ministry of Research, and in part by the European Community's Human Potential Programme under Contract No. HPRN-CT200200320, NANOSPECTRA.
*Electronic address: thierry.melin@isen.iemn.univ-lille1.fr

${ }^{1}$ S. Tiwari, F. Ranah, H. Hanafi, A. Hartstein, E. F. Crabbe, and K. Chan, Appl. Phys. Lett. 68, 1377 (1996).

${ }^{2}$ E. A. Boer, L. D. Bell, M. L. Brongersma, H. A. Atwater, M. L. Ostraat, and R. C. Flagan, Appl. Phys. Lett. 78, 3133 (2001).

${ }^{3}$ T. Mélin, D. Deresmes, and D. Stiévenard, Appl. Phys. Lett. 81, 5054 (2002).

${ }^{4}$ C. Guillemot, P. Budau, J. Chevrier, F. Marchi, F. Comin, C. Alandi, F. Bertin, N. Buffet, Ch. Wyon, and P. Mur, Europhys. Lett. 59, 566 (2002).

${ }^{5}$ D. M. Schaadt, E. T. Yu, S. Sankar, and A. E. Berkowitz, Appl. Phys. Lett. 74, 472 (1999).

${ }^{6}$ T. Mélin, H. Diesinger, D. Deresmes, and D. Stiévenard, Phys. Rev. B 69, 035321 (2004).

${ }^{7}$ Todd D. Krauss and Louis E. Brus, Phys. Rev. Lett. 83, 4840 (1999).

${ }^{8}$ B. Legrand, V. Agache, T. Mélin, J. P. Nys, V. Senez, and D. Stiévenard, J. Appl. Phys. 91, 106 (2002).

${ }^{9}$ T. Mélin, H. Diesinger, D. Deresmes, and D. Stiévenard, Phys. Rev. Lett. 92, 166101 (2004).
${ }^{10}$ The data correspond to the charge injection experiments performed in the work of Ref. 9.

${ }^{11}$ Here the voltage has been applied to the substrate, rather than to the EFM tip, in contrast with all EFM measurement data.

${ }^{12}$ H. Diesinger, T. Mélin, D. Deresmes, and D. Stiévenard, Appl. Phys. Lett. 85, 3546 (2004).

${ }^{13}$ More refined measurements have also been reported in Ref. 12, showing a hysteretic behavior when care was taken to wait until the saturation of the stored charge at a given $V_{i n j}$ voltage and to gradually sweep and cycle the injection voltage in experiments. The hysteresis effect has been assigned to a secondary chargeinjection process associated with surface states on the nanoparticle oxide (Ref. 12). A reminiscence of this effect appears in Fig. 3 from the "noise level" of experimental data.

${ }^{14}$ T. Ando, A. B. Fowler, and F. Stern, Rev. Mod. Phys. 54, 437 (1982).

${ }^{15}$ In fact, calculations for electrons and holes would also require to distinguish between longitudinal and transverse masses for tunneling and kinetic energy purposes. This was not included here. 